\title{
Space-Time Adaptive Interference-Canceling Equalizer for W-CDMA with Known and Unknown Interference
}

\author{
Hsiao-Yung Chen Yumin Lee \\ Dept. of Electrical Engineering and \\ Graduate Institute of Communication Eng., \\ National Taiwan University, Taipei 10617, Taiwan \\ TEL: +886-2-23626508ＦAX: +886-2-23683824Ｅ-mail:yuminlee@cc.ee.ntu.edu.tw
}

\begin{abstract}
Space-time parallel interference cancellation (ST-PIC) is an effective multi-stage multiuser detection scheme for wideband code-division multiple access (W-CDMA). However, the realization of ST-PIC requires accurate channel estimate, a sufficient number of stages, and knowledge of all user's spreading waveforms. With inaccurate channel estimate or insufficient number of stages, the residual multiple access interference (MAI) degrades performance significantly. Furthermore, the performance of ST-PIC is also affected by the presence of interference for which the spreading waveforms are unknown. This paper introduces a new structure for enhancing the performance of ST-PIC in these situations. Simulation results show that a bit error rate (BER) reduction of as much as an order of magnitude is achievable using the proposed structure in the presence of strong MAI.
\end{abstract}

\section{INTRODUCTION}

Direct-sequence code division multiple access (DS-CDMA) is one of the most popular multiple access schemes for current and future wireless communication systems. A DS-CDMA system allows simultaneous transmissions in the same frequency band. Users are differentiated using spreading codes (spreading sequences) that have low cross-correlation. Conventionally, a DS-CDMA receiver uses the RAKE receiver that performs a time correlation operation in alignment with each dispersive delay path to detect the desired information symbols [1]. All other user signals are considered as noise. In general, the performance of this receiver suffers from the multiple access interference (MAI), and if the powers of the users are not controlled properly, strong signals will capture the demodulator in a receiver, resulting in what is known as the near-far problem. Since MAI is the major source of performance of degradation, a DS-CDMA system is an interference-limited system.

Multiuser detection (MUD)[2] is a strategy for jointly detecting mutually interfering signals of information. It has been shown that MUD offers a significant improvement over the conventional RAKE receiver. With advances in hardware technologies, implementation of sophisticated MUD algorithms is becoming more and more feasible. A very effective MUD scheme is a multistage algorithm known as parallel interference cancellation (PIC) [3], in which MAI is estimated and canceled at every stage using symbol decisions from the previous stage. The performance of PIC can be enhanced even more when integrated with transmit and/or receiver antenna diversity [4].

Despite the promising analytical and simulation results, PIC suffers from at least three practical problems. First, just like most MUD schemes, PIC requires an estimate of the propagation channel. Any inaccuracy in the channel estimate results in residual MAI, which can degrade performance significantly. Secondly, even when the channel estimate is very accurate, PIC still requires a sufficient number of stages in order to work properly. Insufficiency in the number of stages due to, e.g., hardware or power constraints, results in residual MAI that degrade performance. Finally, knowledge of every user's spreading waveform is in general required for the PIC, just like most MUD schemes. This information may be unavailable in many situations, e.g., in forward link applications or in the presence of other-cell or other-system interference, making PIC impractical. Interference signals for which the spreading waveforms are unknown are collectively referred to as unknown interference in this paper. Solutions for these problems include the partial PIC (PPIC) [5], adaptive PIC (APIC) [6], and decision-feedback detectors (DFD)[7]. The idea of the PPIC is to remove only part of the MAI at each stage because the estimated MAI is inaccurate. As the MAI estimate becomes more accurate for each successive stage, the amount of removed MAI is also increased. APIC, on the other hand, uses adaptive (usually linear) MAI estimators in each stage, and can be regarded as a more general version of the PPIC. DFD uses a completely different structure for MUD. A DFD includes a feedforward section and a feedback section. The feedforward section cancels the known MAI while the feedback section can reduce the effect of unknown interference as well as residual known MAI. Although the PPIC and APIC are effective and elegant, they still require a sufficient number of stages in order to work properly. On the other hand, the complexity of DFD is significantly higher the PIC, and is difficult to optimize. We therefore propose a structure for enhancing the performance of a space-time PIC (ST-PIC) against unknown interference as well as residual known MAI due to channel estimation error and insufficiency in the number of stages. The key 
component in the proposed structure is an adaptive equalizer referred to as the space-time adaptive interference-canceling equalizer (STAICE). The key idea is that instead of treating the output of ST-PIC as the final detected symbols, a reference is derived to drive the settings of the STAICE towards the "optimal" value so that the effects of signal dispersion caused by the channel, residual known MAI due to channel estimation error and insufficient number of PIC stages, unknown interference, as well as AWGN are mitigated at the same time. Performance improvement achieved by the proposed structure is very significant. Simulation results show that the proposed structure can reduce the bit error rate floor by as much as an order of magnitude in the presence of both known MAI and unknown interference. Although the proposed receiver is simulated only for the synchronous case, it is also applicable to the asynchronous case.

\section{SYSTEM MODEL}

Considering a cellular DS-CDMA system in which signals of a total of $K$ known users are transmitted synchronously and simultaneously over the same frequency band. The continuous-time baseband equivalent transmitted signal of the $k$-th user is expressed as

$$
x_{k}(t)=D_{k}(t) S_{k}(t),
$$

where $D_{k}(t)$ and $S_{k}(t)$ are, respectively, data and spreading waveforms of the $k$-th user. They are defined as ${ }^{1}$

$$
D_{k}(t)=\sum_{m=0}^{\infty} b_{k}[m] p\left(\frac{t-m T}{T}\right)
$$

and

$$
S_{k}(t)=\sum_{m=0}^{\infty} \mathrm{c}_{k}[m] p\left(\frac{t-m T_{c}}{T_{c}}\right),
$$

where $b_{k}[\bullet]$ is the information symbols of the $k$-th user, $T$ and $T_{c}$ are the symbol and chip durations, respectively, $c_{k}[\bullet]$ is the spreading sequence for the $k$-th user, and $p(\bullet)$ is a rectangular pulse shaping function defined as

$$
p(t) \equiv \begin{cases}1, & 0 \leq t<1 \\ 0, & \text { else }\end{cases}
$$

It is assumed that knowledge of the spreading waveforms of these $K$ users is available at the receiver; therefore these $K$ users are referred to as the known users.

At the receiver, we assume that there are a total of $P$ receiving antennas, and the channel between the $k$-th user and $p$-th antenna consists of $L_{k, p}$ resolvable paths. Assume that $\tau_{k, l, p}$ is the arrival time of the $l$-th resolvable path of the channel between the $k$-th user and $p$-th antenna, by the

\footnotetext{
${ }^{1}$ We use square brackets to denote the time index of discrete-time signals.
}

superposition principle the received signal at the $p$-th antenna can be expressed as

$$
r_{p}(t)=\sum_{k=1}^{K} \sum_{l=1}^{L_{k, p}} \alpha_{k, l, p} x_{k}\left(t-\tau_{k, l, p}\right)+i_{p}(t)+n_{p}(t)
$$

where $\alpha_{k, l, p}$ denote the complex gain of the $l$-th resolvable path that takes both channel and antenna gains into account, $i_{p}(t)$ is the unknown interference signal received at the $p$-th antenna, and $n_{p}(\mathrm{t})$ is the additive white Gaussian noise (AWGN) whose two-sided power spectrum density (PSD) is equal to $N_{0} / 2$. The received signals are then processed by the proposed receiver described in the next section.

\section{PROPOSED RECEIVER STRUCTURE}

Figure 1 shows the block diagram of the proposed receiver. The structure is divided into two sub-blocks: the space-time parallel interference canceller (ST-PIC) and space-time adaptive interference-canceling equalizer (STAICE). The ST-PIC partially cancels the MAI generated by known users and provides a somewhat "clean" reference signal for the subsequent STAICE. Using the reference signal, an adaptive algorithm is employed to drive the settings of the STAICE towards the "optimal" value so that the effects of signal dispersion caused by the channel, residual known MAI, unknown interference, and AWGN are mitigated at the same time.

\section{$\underline{\text { ST-PIC }}$}

The initial stage of the ST-PIC consists of a bank of $K$ coherent spatio-temporal RAKE receivers - one for each known user - where $K$ is the number of users with known spreading waveforms as mentioned previously. In this paper, the coherent spatio-temporal RAKE receiver for the $k$-th user is implemented as a bank of $\sum_{p=1}^{P} L_{k, p}$ correlators, with each correlator aligned with one resolvable path between the corresponding user and antenna. The output of the correlators are weighted by the complex conjugates of the estimates of the corresponding path gains, and combined to obtain the output given by

$$
Z_{k}^{(0)}[m]=\sum_{p=1}^{P} \sum_{l=1}^{L_{k, p}} \hat{\alpha}_{k, l, p}^{*} \int_{m T}^{(m+1) T} r_{p}\left(t+\hat{\tau}_{k, l, p}\right) S_{k}^{*}(t) d t .
$$

where $\hat{\alpha}_{k, l, p}$ and $\hat{\tau}_{k, l, p}$ are, respectively, estimates of the path gain $\alpha_{k, l, p}$ and arrival time $\tau_{k, l, p}$. The output in (6) is then sliced to yield

$$
\hat{b}_{k}^{(0)}[m]=D\left(Z_{k}^{(0)}[m]\right),
$$

where $D(\bullet)$ is the appropriate slicing operation. The quantity in (7) is an initial estimate of the symbols transmitted by the $k$-th user, and is used by the subsequent 


\section{ST-PIC stage.}

The ST-PIC used in this paper consists of $S$ stages and is a straightforward extension of the conventional PIC algorithm to the receiver antenna diversity case. In the $s$-th stage $(s=1,2, \ldots, S)$ of the ST-PIC detector, chip-spaced samples of each known user's transmitted signal is first estimated as

$$
\hat{x}_{k}^{(s-1)}[n]=c_{k}[n] \sum_{m=0}^{\infty} \hat{b}_{k}^{(s-1)}[m] u[n-m R],
$$

where $\hat{b}_{k}^{(s-1)}[m]$ is the estimate of the $m$-th symbol transmitted by the $k$-th user obtained in the previous [(s-1)-th] stage of the ST-PIC detector,

$$
R \equiv \frac{T}{T_{c}}
$$

is the spreading factor and is assumed to be an integer, and

$$
u[n] \equiv\left\{\begin{array}{ll}
1, & 0 \leq n<R \\
0, & \text { else }
\end{array} .\right.
$$

The MAI contributed by each known user is then derived from the estimated transmitted signals and estimated path gains and arrival times, and subtracted from the chip-spaced samples of the signal received from each antenna. The result is a set of $K P$ discrete-time signals with reduced MAI given by

$$
\hat{r}_{k, p}^{(s)}[n]=r_{p}\left(n T_{c}\right)-\sum_{\substack{i=1 \\ i \neq k}}^{K} \sum_{l=1}^{L_{i}} \hat{\alpha}_{i, l, p} \hat{x}_{i}^{(s-1)}\left[n-\hat{\tau}_{i, l, p}\right]
$$

for $k=1,2, \ldots, K$ and $p=1,2, \ldots, P$. These signals are next processed by a bank of $K$ discrete-time coherent spatio-temporal RAKE receivers to obtain outputs given by

$$
Z_{k}^{(s)}[m]=\sum_{p=1}^{P} \sum_{l=1}^{L_{k, p}} \hat{\alpha}_{k, l, p}^{*} \sum_{j=m R}^{(m+1) R-1} \hat{r}_{k, p}^{(s)}\left[j+\hat{\tau}_{k, l, p}\right] c_{k}^{*}[j]
$$

for $k=1,2, \ldots, K$. Finally, the output in (12) is sliced to obtain estimates of the symbols transmitted by the $k$-th user, given by

$$
\hat{b}_{k}^{(s)}[m]=D\left(Z_{k}^{(s)}[m]\right),
$$

for the $s$-th stage.

\section{$\underline{\text { STAICE }}$}

The output $\hat{b}_{k}^{(S)}[m]$ of the last ST-PIC stage is the final hard-decision output of a conventional ST-PIC. Various studies [3-7] in the literature have shown that very good performance is achievable using a conventional ST-PIC with sufficient number of stages when the spreading waveforms all users are known and an accurate estimate of the channel is available. However, when the number of ST-PIC stages is insufficient, or when the channel estimate is inaccurate, the resulting residual MAI from known users degrade the performance of ST-PIC significantly. Furthermore, the performance of the conventional ST-PIC is also affected by the presence of unknown interference. To improve the performance of the ST-PIC in these situations, we enhance the conventional ST-PIC with the space-time adaptive interference-canceling equalizer (STAICE).

The STAICE is shown in Figure 2 for one particular user for $P=2$. The key idea is that instead of treating the output of the last ST-PIC stage as the final hard-decision output, we form signals $\hat{x}_{k}^{(S)}[n], k=1, \ldots, K$, according to (8), and use them as reference signals to drive a bank of $K P$ adaptive equalizers (one for each user on each antenna). Each adaptive equalizer is a linear finite impulse response (FIR) filter with $N_{D}$ chip-spaced coefficients adapted at the chip-rate using the normalized least-mean-square (NLMS) algorithm[8]. NLMS is chosen here because of its simplicity and robustness against eigenvalue spread of the correlation matrix of the input signal vector [8]. The input of the adaptive equalizers for the $k$-th user on the $p$-th antenna is the signal $\hat{r}_{k, p}^{(S)}[n]$ given in (11), and the corresponding output is given by

$$
\hat{r}_{k, p}[n]=\sum_{j=0}^{N_{D}-1} w_{k, p}^{*}[n, j] \hat{r}_{k, p}^{(S)}[n-j]
$$

where $w_{k, p}{ }^{*}[n, j]$ is the $j$-th filter coefficient at time $n$. The outputs of the $P$ equalizers for the $k$-th user are then linearly combined to give a single output given by

$$
\hat{r}_{k}[n]=\sum_{p=1}^{P} \frac{\hat{r}_{k, p}[n]}{<\left|e_{k, p}[n]\right|^{2}>},
$$

where $\left\langle\left|e_{k, p}[n]\right|^{2}>\right.$ is the linearly smoothed magnitude-square of the error defined as

$$
e_{k, p}[n]=\hat{r}_{k, p}[n]-\hat{x}_{k}^{(S)}[n] .
$$

Finally, $\hat{r}_{k}[n]$ is despreaded using the spreading sequence of the $k$-th user to obtain

$$
Z_{k}[m]=\sum_{j=m R}^{(m+1) R-1} \hat{r}_{k}[j] c_{k}^{*}[j],
$$

which is then sliced to obtain the final output

$$
\hat{b}_{k}[m]=D\left(Z_{k}[m]\right) \text {. }
$$

Several points are worth mentioning regarding the proposed structure. First, on the $p$-th antenna, the input signal $\hat{r}_{k, p}^{(S)}[n]$ of the STAICE, given in (11), consists of the $k$-th user's signal distorted by the multipath fading channel, the residual MAI from known users, unknown intereference, and AWGN. On the other hand, the reference signal $\hat{x}_{k}^{(S)}[n]$ of the STAICE given in (8) is an estimate of the transmitted signal of the $k$-th user. It can thus be seen that the STAICE plays the role of a chip-rate equalizer that takes signal dispersion, residual known MAI, unknown interference, and AWGN jointly into account. Although the statistics of the residual known MAI and unknown intereference are not exactly known to the receiver, the NLMS algorithm can drive 
the coefficients of the STAICE towards the "optimal" settings provided that $\hat{x}_{k}^{(S)}[n]$ reasonably resembles the actual transmitted signal $x_{k}[n]$. Secondly, $\hat{x}_{k}^{(S)}[n]$ is obviously different from $x_{k}[n]$ because $\hat{x}_{k}^{(S)}[n]$ is derived from the detected symbols $\hat{b}_{k}^{(S)}[m]$, which inevitably contain errors due to the residual known MAI, unknown interference, and AWGN. However, as will be shown later by simulation, even when $\hat{b}_{k}^{(S)}[m]$ contains too many errors to be useful directly, $\hat{x}_{k}^{(S)}[n]$ still resembles $x_{k}[n]$ enough to be a useful reference signal for the adaptive algorithm. In this case the performance can be significantly improved by the STAICE, as will be shown by simulation. Thirdly, the linear combining in (13) is similar to maximal ratio combining (MRC) in the sense that the combining weight is inversely proportional to the estimation error power. It may be argued that by jointly adapting the $P$ equalizers for the $k$-th user and simply adding their outputs, possibly better performance may be achievable. However, preliminary simulation results show that the performance of the proposed scheme is at least as good as the joint adaptation scheme. One plausible explanation is that the dimensionality of the coefficient vector is larger for joint adaptation, thus requiring training signals of better quality and larger quantity. Finally, the assumption of synchronous DS-CDMA is made only to simplify the modeling and simulation of the ST-PIC. This assumption is not used for the STAICE, therefore the STAICE can also be used for enhancing the performance of MUD for asynchronous DS-CDMA.

\section{SIMULATION RESULTS}

The performance of the proposed receiver is evaluated by simulating a total of 10 known users $(K=10)$ in a synchronous DS-CDMA system with dual antenna reception $(P=2)$. In the simulations, the transmitter is implemented according to the W-CDMA unplink technical specifications [9]. Each known user has one dedicated physical data channel (DPDCH) and is assigned a channelization orthogonal variable spreading factor (OVSF) code with spreading factor (SF) equal to $R=64$. A total of 2000 bursts of random user data are simulated, where each burst is 20 frames long and each frame has a length ${ }^{2}$ of 38400 chip-periods (10ms), as defined in the W-CDMA specifications. The scrambling code for each user is the short scrambling code with different initial states, as specified in the W-CDMA specifications. The number of pilot bits in each time-slot is equal to $8\left(N_{\text {pilot }}=8\right)$. The modulation is binary phase shift keying (BPSK). Perfect power control is assumed for the known users so that each user has the same average received energy.

\footnotetext{
${ }^{2}$ For simplicity, we assume that mobile units use all 15 time-slots in a frame.
}

Unless otherwise stated, the wireless channel is modeled as a Gaussian wide-sense stationary uncorrelated scattering (GWSSUS) multipath propagation channel corrupted by AWGN and zero or two unknown interferers. A two-path power-delay profile is assumed, where the relative delay between the two paths is 4 chip-periods and relative power levels of the two paths are 0 and $-10 \mathrm{~dB}$, respectively. It is also assumed that there is no line-of-sight component in the multipath propagation channel and that the channel stays approximately constant during each burst, but is uncorrelated from burst to burst.

At the receiver, it is assumed that the number $L_{k, p}$ and arrival time $\tau_{k, l, p}$ of each user's propagation paths are known. Estimates $\hat{\alpha}_{k, l, p}$ of the path gains are then obtained using either the least-squares (LS) or turbo multiuser space-time channel estimators previously proposed in [10]. The received signal is then processed by the ST-PIC and STAICE in the proposed receiver structures as previously described. The performance of the conventional (ST-PIC-only) receiver is also simulated as a baseline for comparison. In the simulation results, the number of stages in the ST-PIC is set to $S=1$, and the number of coefficients for STAICE is set to $N_{D}=8$.

The performance of the conventional (ST-PIC-only) and proposed receivers (ST-PIC enhanced by STAICE) is shown in Figure 3 for the AWGN channel with no unknown interference. The average bit error rates (BER) of both the LS and turbo channel estimators are plotted in this figure as functions of $E_{b} / N_{0}$, where $E_{b}$ is the transmitted energy per bit. For the ST-PIC, it can be seen that the BER curves saturate at high $E_{b} / N_{0}$ as expected. Here the performance is dominated by the large amount of residual known MAI because only one stage $(S=1)$ is used in the ST-PIC. It can also be seen that the performance of the turbo channel estimator is significantly better than the LS channel estimator for the ST-PIC, which is consistent with the result reported in [10]. The turbo channel estimator provides more accurate channel estimates, thus reducing the effect of residual known MAI. Finally, it can be seen that the proposed structure significantly improves the performance of the ST-PIC for both types of channel estimators. The BER floor of the proposed receiver is 5 to 10 times lower than the ST-PIC. These results indicate that, at least for the AWGN channel without unknown interference, the proposed STAICE can suppress residual known MAI and provide significant performance enhancement, even though its reference signals contain errors.

The performance of the proposed receiver is next evaluated for the two-path GWSSUS channel with and without unknown interference. The average BER of the proposed receiver and ST-PIC are plotted as functions of $E_{b} / N_{0}$ in Figure 4 using the LS channel estimator. The curves labeled with "ST-PIC/UI" and "Proposed/UI" are obtained assuming the presence of two unknown interferers. 
These unknown interferers transmit at the same power levels as the known interferers, however the receiver does not know their spreading waveforms. It can be seen that the presence of unknown interference indeed significantly affects the performance of both the ST-PIC and proposed receivers. However, the performance of the proposed receiver is still significantly better than the ST-PIC regardless of the number of unknown interferers. In particular, the BER floor of the proposed receiver is again 5 to 10 times lower than the ST-PIC. These results again verify the ability of the proposed STAICE to suppress residual known MAI and unknown interference and enhance performance for the two-path fading channel with and without unknown interference.

Similar performance curves are shown in Figure 5 using the turbo channel estimator. Comparing this figure to Figure 4, one can see that the performance of the turbo channel estimator is significantly better than the LS estimator for all cases simulated, just as for the AWGN channel. Furthermore, the performance of the proposed receiver is also significantly better than the ST-PIC regardless of the number of unknown interferers, just as in Figure 4. The performance improvement is not as large, however, because here the effect of residual known MAI is much less because a better channel estimator is used for the ST-PIC. However the ability of the proposed STAICE to suppress residual known MAI and unknown interference is still evident from Figure 5.

\section{CONCLUSION}

The performance of the space-time parallel interference canceller (ST-PIC) is affected by residual MAI caused by channel estimation error and insufficiency in the number of PIC stages, unknown interference, and AWGN. The space-time adaptive interference-canceling equalizer (STAICE) is proposed for enhancing the performance of the ST-PIC) against these impairments. By using the output of ST-PIC to derive a reference signal for the adaptation algorithm, the STAICE can indeed achieve significant performance gains in the presence of strong MAI. Although only the synchronous case is simulated, the proposed STAICE is equally applicable to the asynchronous case.

\section{REFERENCES}

[1] J. G. Proakis, Digital Communications, 3rd ed., pp. McGraw Hill, Inc., 1995.

[2] S. Verdu, Multiuser Detection, Cambridge Univ. Press, 1998.

[3] M. K. Varanasi and B. Aazhang, "Multistage detection in asynchronous code-division multiple-access communications," IEEE Trans. Commun., vol. 38, pp. 509-519, Apr. 1990.

[4] J. Weng et. al., "Multistage Interference Cancellation with Diversity Reception for Asynchronous QPSK DS/CDMA Systems over Multipath Fading Channels," IEEE J. on Sel. Areas in Comm., Vol. 17, No. 12, pp. 2162 - 80, December 1999.

[5] D. Divsalar et. al., "Improved Parallel Interference Cancellation for CDMA," IEEE Trans. On Comm., Vol. 46, pp. 258 - 68, February 1998.

[6] G. Xue, et. al., "Adaptive Multistage Parallel Interference Cancellation for CDMA," IEEE J. Select. Areas Commun., vol. 17, pp. 1815-1827, Oct. 1999.

[7] A. Duel-Hallen, "A Family of Multiuser Decision-Feedback Detectors for Asynchronous CDMA Channels," IEEE Trans. On Commun., Vol. 43, pp. 421 - 34, Feb./Mar./Apr. 1995.

[8] S. Haykin, Adaptive Filter Theory, Prentice Hall, 3rd ed., 1996.

[9] $3^{\text {rd }}$ Generation Partnership Project: Technical Specification 3GTS 25.213 V. 4.1.0, June 2001.

[10] M. C. Tournier et. al., "Direct and Turbo Multi-User Spatio-Temporal Channel Estimation Schemes for CDMA FDD UMTS Uplink," IEEE ICASSP '00, Vol. 5, pp. 2805-2808, Jun. 2000.

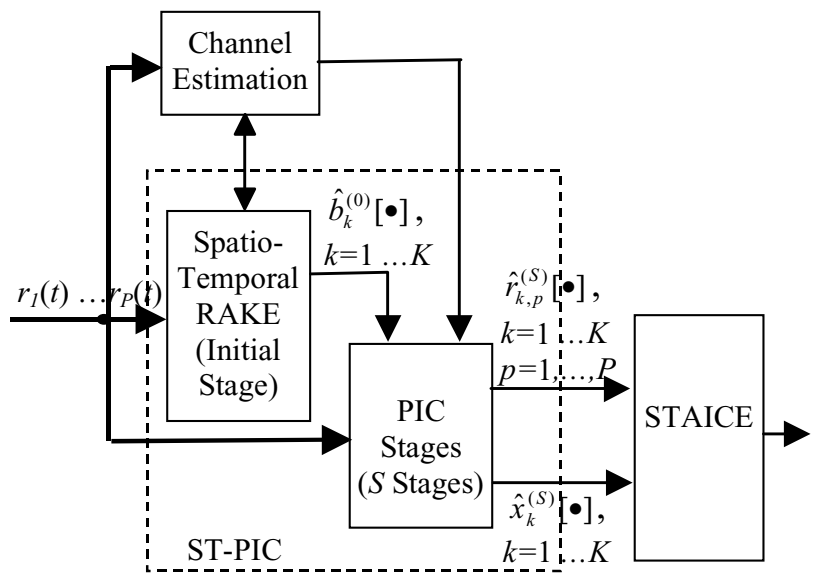

Fig. 1. The proposed receiver structure.

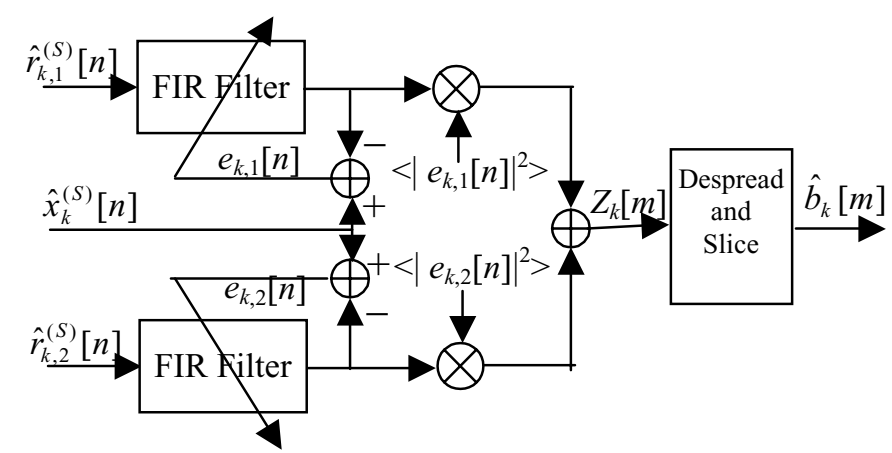

Fig. 2. Block diagram of STAICE for one particular user for $P=2$. 


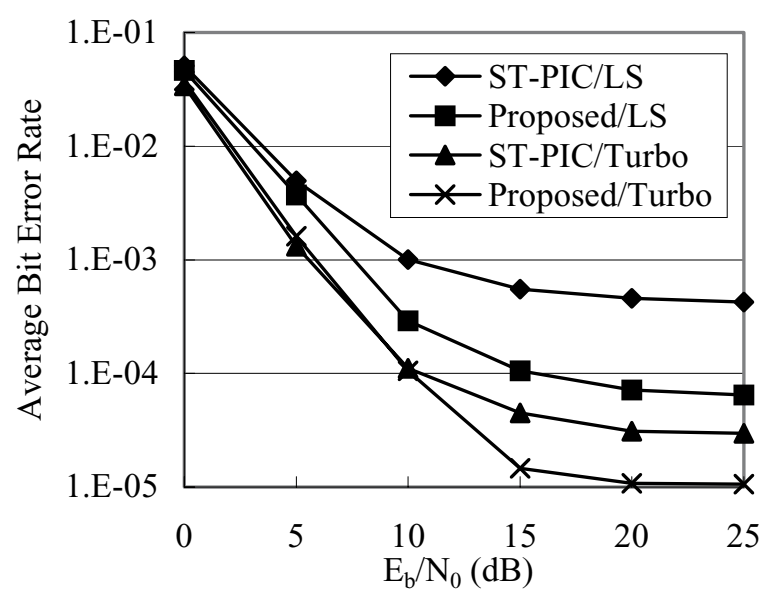

Fig. 3. BER of the proposed receiver for the AWGN channel without unknown interference. The number of known users is 10 .

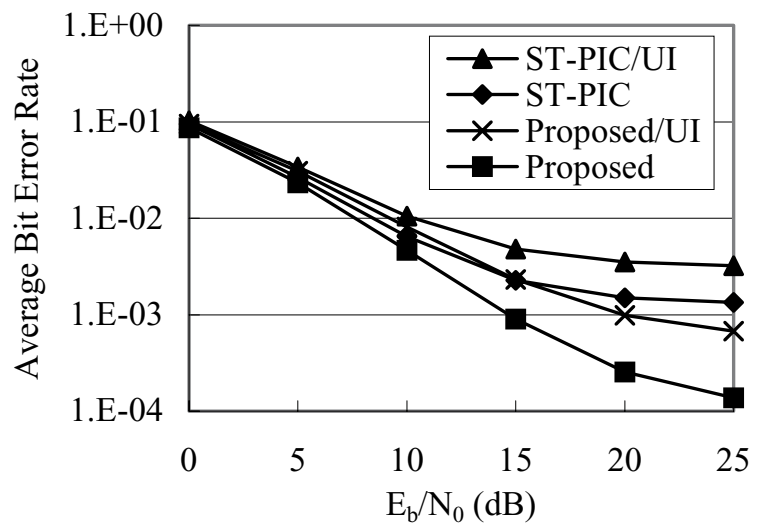

Fig. 4. BER of the proposed receiver for the two-path fading channel with and without unknown interference. LS channel estimator is used.

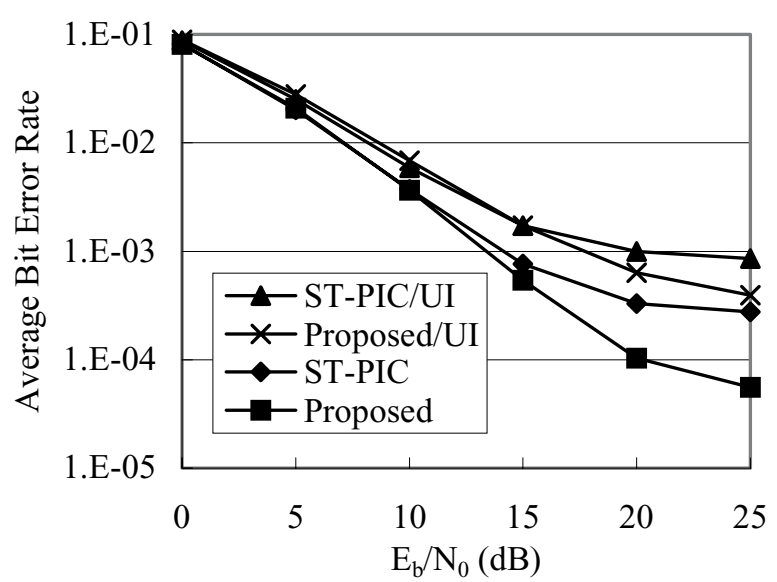

Fig. 5. BER of the proposed receiver for the two-path fading channel with and without unknown interference. The turbo channel estimator is used. 